\title{
Robust constrained waveform design for MIMO radar with uncertain steering vectors
}

\author{
Xianxiang Yu${ }^{1}$, Guolong Cui ${ }^{*}$, Marco Piezzo ${ }^{2}$, Salvatore lommelli ${ }^{3}$ and Lingjiang Kong ${ }^{1}$
}

\begin{abstract}
This paper considers the robust waveform design of multiple-input multiple-output (MIMO) radar to enhance targets detection in the presence of signal-dependent interferences assuming the knowledge of steering vectors is imprecise Specifically, resorting to semidefinite programming (SDP)-related technique, we first maximize the worst-case signal-to-interference-plus-noise ratio (SINR) over uncertain region to optimize waveform covariance matrix forcing a uniform elemental power requirement. Then, based on least square (LS) approach, we devise the waveform accounting for constant modulus and similarity constraints by the obtained waveform covariance matrix using cyclic algorithm (CA). Finally, we assess the effectiveness of the proposed technique through numerical simulations in terms of non-uniform point-like clutter and uniform clutter.
\end{abstract}

Keywords: Robust waveform design, Multiple-input multiple-output (MIMO), Signal-dependent interferences, Signal-to-interference-plus-noise ratio (SINR), Waveform covariance matrix, Constant modulus and similarity constraints

\section{Introduction}

Recently, some advances in radar technology including digital arbitrary waveform generators, solid state transmitters, and high-speed and off-the-shelf processors [1-3] have been greatly developed, making possible for modern radar systems to adaptively adjust the synthesized transmit waveform to detect environment. This adjustment of transmit waveform can be used to significantly enhance its ability of target detection, identification, and classification. Nevertheless, usually, these techniques suffer from inaccuracies on the knowledge of the actual target/clutter processes, which is necessary to ensure an effective adaptation.

Robust waveform design to resist uncertainty sets of target or clutter parameters (i.e., Doppler frequency, angle) has received considerable attention during the last decades [4-19]. According to the structure of radar systems, these works can be classified into two categories. The first one focuses on the robust design for monostatic radar systems. Specifically, in [4], the design of robust radar code under energy and similarity constraints

*Correspondence: cuiguolong@uestc.edu.cn

'School of Electronic Engineering, University of Electronic Science and Technology of China, 2006 Xi Yuan Ave, West Hi-tech Zone, Chengdu, China

Full list of author information is available at the end of the article is investigated to improve the worst-case signal-to-noise ratio (SNR) over the possible target Doppler frequencies by using semidefinite programming (SDP)-related technique. In [5], the robust approach based on SDP and randomization with respect to the target Doppler is proposed to synthesize the radar waveform accounting for a peak-to-average-power ratio (PAR) and an energy constraint in order to the improvement of the worst-case SNR. For the detection problem of the extended target with the uncertain set on target impulse response (TIR), the robust transmit waveform accounting for constant modulus constraint and receiving filter have been designed jointly. In [6], considering worst-case SINR as the performance measure and assuming an interval of target Doppler shift are available, the robust design of transmit sequence and receive filter under energy and similarity constraints has been considered by exploiting related SDP relaxation. Considering the same criteria, the uncertain set, and waveform constraints, the related generalized Dinkelbach's procedure [7] is developed to optimize radar waveform and doppler filter bank. For extended targets considering the uncertainties on the TIR and using the PAR and energy as signal constraints, in [8], a design procedure, based on SDP relaxation and randomization technique, is proposed to devise robust transmit 
code and receive filter aiming to improve the worst-case SINR. Assuming as figure of merit worst-case SINR, in [9], a max-min approach against the uncertainly sets of TIR for extended targets and the second-order statistics of the interference, is exploited to devise the waveform considering energy constraint.

The second category focuses on the robust waveform design for MIMO radar systems. Precisely, in [10], based on the criteria of the mutual information (MI) and minimum mean-square error (MMSE) estimation, the minimax robust waveform design has been addressed by leveraging the a priori knowledge of target power spectral density lying in an uncertainty class of spectrabounded and signal-independent interference statistics. In [11], assuming as figure of merit the cumulated power of probing signal, the worst-case scenario against the uncertain sets of targets locations have been considered to design the waveform covariance matrix forcing a uniform elemental power constraint into each transmitting antenna. In [13], a robust approach against uncertainties on steering vectors is proposed to design the robust transmit beampattern so as to minimize beampattern sidelobes considering power constraint and $3 \mathrm{~dB}$ mainbeam width constraint. In [14], using the imperfect clutter prior knowledge, the robust waveform design has been addressed for maximizing the worst-case SINR. The robust joint design problem of the space-time transmit code (STTC) and the space-time receive filter (STRF) for a moving point-like target is considered in [15] assuming as figure of merit the worst-case SINR over the actual and signal-dependent clutter statistics and considering both energy and similarity constraints on the sought code. In order to improve the worst-case output SINR over the unknown angle of the target of interest, the robust design problem of transmit waveform satisfying energy constraint and the receive filter is investigated under signal-dependent interferences [16]. In particular, we here note that the signal-dependent interferences are from the terrain and the objects of no tactical importance within the illuminated area, generated by the reflections of the signal and transmitted by the radar of interest [17]. In other words, this is a kind of self-induced radar interference, usually referred to as the reverberation phenomenon, owing to the interaction of the transmitted waveform with the scattering environment. These interferences would severely impair the target detectability of radar systems. In [18], based on the worst-case SNR over the uncertain on steering vector, an iterative algorithm is presented to optimize the waveform covariance matrix under a power constraint. In [19], assuming either the peak sidelobe level (PSL) or the integrated sidelobe level (ISL) as figure of merit, the robust waveform covariance matrixes design has been addressed for the purpose of optimizing the worst-case transmit beampattern over steering mismatches accounting for power constraint and $3 \mathrm{~dB}$ main-beam width constraint.

In this paper, we still focus on the robust waveform design of MIMO radar considering practical constraints for enhancing target detectability in the presence of signal-dependent interferences. Precisely, in order to improve the worst-case SINR over the uncertain sets of the steering vectors, we first synthesize waveform covariance matrix accounting for a uniform power constraint by resorting to SDP-related technique. Then, based on least square (LS) approach, we exploit cyclic algorithm (CA) to design waveform under constant modulus and similarity constraints so as to approximate the obtained waveform covariance matrix. Finally, at the analysis stage, we consider two scenarios of non-uniform point-like clutter and uniform clutter to evaluate the performance of the proposed devise procedure. Results exhibit that the proposed algorithm has the capability of ensuring an improved worst-case performance.

The remainder of the paper is organized as follows. In Section 2, we show the system model. In Section 3, we consider the robust design of waveform covariance matrix. In Section 4, we consider the waveform synthesis under some practical constraints. In Section 5, we evaluate the performance of the proposed procedure. Finally, in Section 6, we provide concluding remarks and possible future research tracks.

\subsection{Notation}

We adopt the notation of using boldface for vectors $\boldsymbol{a}$ (lower case) and matrices $\boldsymbol{A}$ (upper case). $\|\boldsymbol{A}\|$ denotes the two-norm of $\boldsymbol{A}$. The transpose, the conjugate, and the conjugate transpose operators are denoted by the symbols $(\cdot)^{T},(\cdot)^{*}$, and $(\cdot)^{\dagger}$ respectively. $\operatorname{tr}(\cdot)$ denotes the trace of square matric. $\boldsymbol{I}_{N}$ denotes $N \times N$-dimensional identity matrix. $\mathbb{C}^{N}$ and $\mathbb{H}^{N}$ are respectively the sets of $N$-dimensional vectors of complex numbers and $N \times N$ Hermitian matrices. The curled inequality symbol $\succeq$ (and its strict form $\succ$ ) is used to denote generalized matrix inequality: for any $\boldsymbol{A} \in \mathbb{H}^{N}, \boldsymbol{A} \succeq \mathbf{0}$ means that $\boldsymbol{A}$ is a positive semidefinite matrix ( $\boldsymbol{A} \succ \mathbf{0}$ for positive definiteness).

The vec $(\boldsymbol{A})$ denotes the column vector obtained by stacking the columns of $\boldsymbol{A}$. The letter $j$ represents the imaginary unit (i.e., $j=\sqrt{-1}$ ). For any complex number $x$, we use $\Re(x)$ respectively the real part of $x$. In addition, $|x|$ and $\arg (x)$ represent respectively the modulus and the argument of $x . \mathbb{E}[\cdot]$ denotes statistical expectation.

\section{System model}

We consider a colocated narrow band MIMO radar system with $N_{T}$ transmitting antennas and $N_{R}$ receiving antennas. Each transmitting antenna emits a distinct waveform $s_{n}(m), n=1,2, \cdots, N_{T}, m=1,2, \cdots, M$, with $M$ being the sample number of each transmitting pulse. 
Let us denote by $\boldsymbol{s}(n)=\left[\boldsymbol{s}_{1}(n), \boldsymbol{s}_{2}(n), \cdots, \boldsymbol{s}_{N_{T}}(n)\right]^{T} \in \mathbb{C}^{N_{T}}$, the $n$th sample of the $N_{T}$ waveforms. At each receiver, the received waveform is down-converted to baseband, undergoes a pulse-matched filtering operation, and then is sampled. Hence, the observations of the $n$th sample for a far-field target at the azimuth angle $\theta_{0}$ can be expressed as

$$
\boldsymbol{x}(n)=\alpha_{0} \boldsymbol{A}\left(\theta_{0}\right) \boldsymbol{s}(n)+\boldsymbol{d}(n)+\boldsymbol{v}(n)
$$

where

- $\alpha_{0}$ is a complex parameter accounting for the target radar cross section (RCS), channel propagation effects, and other terms involved into the radar range equation.

- $\mathbf{A}(\theta)=\boldsymbol{a}_{r}^{*}(\theta) \boldsymbol{a}_{t}^{\dagger}(\theta)$, in which for the azimuth angle $\theta, \boldsymbol{a}_{t}(\theta)$ and $\boldsymbol{a}_{r}(\theta)$ denote, respectively, the transmit spatial steering vector and the receive spatial steering vector. In particular, for the uniform linear arrays (ULAs), they are given by

$$
\begin{gathered}
\boldsymbol{a}_{t}(\theta)=\frac{1}{\sqrt{N_{T}}}\left[1, e^{j 2 \pi \frac{d_{T}}{\lambda} \sin \theta}, \ldots, e^{j 2 \pi \frac{d_{T}}{\lambda}\left(N_{T}-1\right) \sin \theta}\right]^{T}, \\
\boldsymbol{a}_{r}(\theta)=\frac{1}{\sqrt{N_{R}}}\left[1, e^{j 2 \pi \frac{d_{R}}{\lambda} \sin \theta}, \ldots, e^{j 2 \pi \frac{d_{R}}{\lambda}\left(N_{R}-1\right) \sin \theta}\right]^{T}
\end{gathered}
$$

with $d_{T}$ and $d_{R}$, respectively, the array inter-element spacing of the transmitter and the receiver.

- $\boldsymbol{d}(n) \in \mathbb{C}^{N_{R}}, n=1,2, \cdots, M$, accounts for $K$ signal-dependent uncorrelated point-like interfering scatterers. Specifically, considering the $k$ th interfering source located at $\theta_{k}, k=1,2, \cdots, K$, the received interfering vector $\boldsymbol{d}(n)$ can be expressed as the superposition of the returns from $K$ interference sources, i.e.,

$$
\boldsymbol{d}(n)=\sum_{k=1}^{K} \rho_{k} \mathbf{A}\left(\theta_{k}\right) \boldsymbol{s}(n),
$$

with $\rho_{k}$ being the complex amplitude of the $m$ th interferences.

- $\boldsymbol{v}(n) \in \mathbb{C}^{N_{R}}, n=1,2, \cdots, M$, denotes additive noise, modeled as independent and identically distributed (i.i.d.) complex circular zero-mean Gaussian random vector, i.e., $\boldsymbol{v}(n) \sim \mathcal{C N}\left(0, \sigma_{v}^{2} \mathbf{I}_{N_{R}}\right)$.

\section{Robust waveform covariance matrix design}

In this section, we formalize the problem of the design of robust waveform covariance matrix in order to maximize the worst-case output SINR criterion under specific practical constraints. Finally, we provide the related SDP technique to solve the considered problem.

\subsection{Output SINR}

In this subsection, for signal-independent interferences, we derive the expression of SINR with respect to waveform covariance matrix $\boldsymbol{R}$. Specifically, according to the signal model (1), the received useful power can be computed as

$$
\begin{aligned}
& \frac{1}{M} \sum_{m=1}^{M} \mathbb{E}\left[\left\|\alpha_{0} \mathbf{A}\left(\theta_{0}\right) \boldsymbol{s}(m)\right\|^{2}\right] \\
& =\frac{1}{M} \mathbb{E}\left[\left\|\alpha_{0}\right\|^{2}\right] \sum_{m=1}^{M} \boldsymbol{s}^{\dagger}(m) \boldsymbol{a}_{t}(\theta) \boldsymbol{a}_{r}^{T}\left(\theta_{0}\right) \boldsymbol{a}_{r}^{*}\left(\theta_{0}\right) \boldsymbol{a}_{t}^{\dagger}\left(\theta_{0}\right) \boldsymbol{s}(m) \\
& =\mathbb{E}\left[\left\|\alpha_{0}\right\|^{2}\right] \operatorname{tr}\left(\boldsymbol{a}_{t}^{\dagger}\left(\theta_{0}\right) \sum_{m=1}^{M} \boldsymbol{s}(m) \boldsymbol{s}^{\dagger}(m) \boldsymbol{a}_{t}\left(\theta_{0}\right)\right) \\
& =\mathbb{E}\left[\left\|\alpha_{0}\right\|^{2}\right] \boldsymbol{a}_{t}^{\dagger}\left(\theta_{0}\right) \boldsymbol{R a}_{t}\left(\theta_{0}\right)
\end{aligned}
$$

where $\boldsymbol{R}=\frac{1}{M} \sum_{m=1}^{M} \boldsymbol{s}(m) \boldsymbol{s}^{\dagger}(m)$ stands for the waveform covariance matrix. Similarly, since the scatterers are uncorrelated, the received disturbance power is given by

$$
\begin{aligned}
& \frac{1}{M} \sum_{m=1}^{M} \mathbb{E}\left[\left\|\sum_{k=1}^{K} \alpha_{k} \mathbf{A}\left(\theta_{k}\right) \boldsymbol{s}(m)\right\|^{2}\right] \\
& =\sum_{k=1}^{K} \mathbb{E}\left[\left\|\alpha_{k}\right\|^{2}\right] \boldsymbol{a}_{t}^{\dagger}\left(\theta_{k}\right) \boldsymbol{R} \boldsymbol{a}_{t}\left(\theta_{k}\right) .
\end{aligned}
$$

Interestingly, inspection of (3) and (4) exhibits that the receiving steer vectors have no effect on the useful power as well as the interfering power. In addition, we also observe that the useful power functionally depends on waveform covariance matrix $\boldsymbol{R}$ so does the clutter power.

Finally, on the basis of the above equations, the SINR can be defined as

$$
\rho(\boldsymbol{R})=\frac{\delta_{0} \boldsymbol{a}_{t}^{\dagger}\left(\theta_{0}\right) \boldsymbol{R} \boldsymbol{a}_{t}\left(\theta_{0}\right)}{\sum_{k=1}^{K} \delta_{k} \boldsymbol{a}_{t}^{\dagger}\left(\theta_{k}\right) \boldsymbol{R} \boldsymbol{a}_{t}\left(\theta_{k}\right)+\sigma^{2}},
$$

with $\delta_{k}=\mathbb{E}\left[\left\|\alpha_{k}\right\|^{2}\right]$, where $\sigma^{2}$ denotes the noise power. We notice that the objective function $\rho(\boldsymbol{R})$ requires the explicit knowledge of the steer vectors $\boldsymbol{a}_{t}\left(\theta_{k}\right), k=$ $0,1, \cdots, K$. However, from a practical point of view, the exact knowledge of $\boldsymbol{a}_{t}\left(\theta_{k}\right)$ can not be available. Hence, in the next subsection, some practical constraints are considered to overcome this drawback.

\subsection{Uncertain sets of steering vectors and power constraint}

In practical considerations, due to array non-idealities and imperfect calibration, the actual steering vector can not be exactly known. To this end, we enforce some quadratic 
constraints into $\boldsymbol{a}_{k}, k=0,1, \cdots, K$, (where we write $\boldsymbol{a}_{t}\left(\theta_{k}\right)$ into $\boldsymbol{a}_{k}$ for simplifying the notations) i.e.,

$$
\begin{aligned}
\mathcal{A}_{k}= & \left\{\boldsymbol{a}_{k} \mid 0 \leq \boldsymbol{a}_{k}^{\dagger} \boldsymbol{B}_{k m} \boldsymbol{a}_{k}+2 \Re\left(\boldsymbol{b}_{k m}^{\dagger} \boldsymbol{a}_{k}\right)+b_{k m} \leq u_{k m},\right. \\
& m=1,2\}, k=0,1, \cdots, K
\end{aligned}
$$

where $\boldsymbol{B}_{k m} \in \mathbb{H}^{N_{T}}, \boldsymbol{b}_{k m} \in \mathbb{C}^{N_{T}}$, and $b_{k m}, u_{k m}$ both are real values for $m=1,2, k=0,1, \cdots, K$. We remark that generalized similarity and conical and norm constraints are the special cases of (6). In particular, the detailed illumination of these constraints can be obtained in [19].

Furthermore, to control the amount of transmitted power, we force a uniform power constraint in each transmitting antenna, i.e.,

$$
\mathcal{R}_{u}=\left\{\boldsymbol{R} \in \mathbb{H}^{N_{T}} \mid \boldsymbol{R} \geq 0, \boldsymbol{R}(i, i)=c, i=1,2, \cdots, N_{T}\right\},
$$

where $c$ is the transmitted power of each emitter.

\subsection{Waveform covariance matrix design problem}

Based on the aforementioned discussion, the robust design problem of waveform covariance matrix $\boldsymbol{R}$ to optimize the worst-case SINR over mismatching steer vectors can be summarized as follows:

$$
\max _{\boldsymbol{R} \in \mathcal{R}_{u}}\left[\min _{\boldsymbol{a}_{k} \in \mathcal{A}_{k}, k \in \mathcal{K}} \frac{\delta_{0} \boldsymbol{a}_{0}^{\dagger} \boldsymbol{R} \boldsymbol{a}_{0}}{\sum_{k=1}^{K} \delta_{k} \boldsymbol{a}_{k}^{\dagger} \boldsymbol{R} \boldsymbol{a}_{k}+\sigma^{2}}\right],
$$

where $\mathcal{K}=\{0,1, \cdots, K\}$. (8) is in general NP-hard which has no closed-form solution.

\subsection{Waveform covariance matrix design algorithm}

In this subsection, we focus on studying the solution of (8) by using SDR-related technique. Before proceeding further, we first transform (8) as follows:

$$
\max _{\boldsymbol{R} \in \mathcal{R}_{u}}\left[\frac{\min _{\boldsymbol{a}_{0} \in \mathcal{A}_{0}} \delta_{0} \boldsymbol{a}_{0}^{\dagger} \boldsymbol{R} \boldsymbol{a}_{0}}{\sum_{k=1}^{K} \max _{\boldsymbol{a}_{k} \in \mathcal{A}_{k}} \delta_{k} \boldsymbol{a}_{k}^{\dagger} \boldsymbol{R} \boldsymbol{a}_{k}+\sigma^{2}}\right] .
$$

Interestingly, we observe that (9) can be solved by exploiting SDR technique [19]. Specifically, after the same line of reasoning as [19], this problem is tantamount to SDP problem given by

$$
\begin{aligned}
& \begin{cases}\max _{\bar{R}, t, t_{1}} & t_{1} \\
\text { s.t. } & u_{01} x_{01}+u_{02} x_{02}+x_{03} \geq t_{1}\end{cases} \\
& \sum_{k=1}^{K} u_{k 1} y_{k 1}+u_{k 2} y_{k 2}+y_{k 3} \geq-1 \\
& \overline{\boldsymbol{R}}(i, i)=c t, i=1,2, \cdots, N_{T} \\
& \left(\begin{array}{cc}
\delta_{0} \overline{\boldsymbol{R}} & \mathbf{0} \\
\mathbf{0} & -x_{03}
\end{array}\right)-\left(x_{01}+w_{01}\right)\left(\begin{array}{ll}
\boldsymbol{B}_{01} & \boldsymbol{b}_{01} \\
\boldsymbol{b}_{01}^{\dagger} & b_{01}
\end{array}\right) \\
& -\left(x_{02}+w_{02}\right)\left(\begin{array}{ll}
\boldsymbol{B}_{02} & \boldsymbol{b}_{02} \\
\boldsymbol{b}_{02}^{\dagger} & b_{02}
\end{array}\right) \succeq \mathbf{0}, \\
& -\left(\begin{array}{cc}
\delta_{k} \overline{\boldsymbol{R}} & \mathbf{0} \\
\mathbf{0} & y_{k 3}
\end{array}\right)-\left(y_{k 1}+z_{k 1}\right)\left(\begin{array}{cc}
\boldsymbol{B}_{k 1} & \boldsymbol{b}_{k 1} \\
\boldsymbol{b}_{k 1}^{\dagger} & b_{k 1}
\end{array}\right) \\
& -\left(y_{k 2}+z_{k 2}\right)\left(\begin{array}{ll}
\boldsymbol{B}_{k 2} & \boldsymbol{b}_{k 2} \\
\boldsymbol{b}_{k 2}^{\dagger} & b_{k 2}
\end{array}\right) \succeq \mathbf{0}, k=1,2, \cdots, K, \\
& \overline{\boldsymbol{R}} \succeq \mathbf{0}, t \geq 0, t_{1} \in \mathbb{R} \text {, } \\
& x_{01} \leq 0, x_{02} \leq 0, x_{03} \in \mathbb{R}, w_{01} \geq 0, w_{02} \geq 0 \\
& y_{k 1} \leq 0, y_{k 2} \leq 0, y_{k 3} \in \mathbb{R}, z_{k 1} \geq 0, z_{k 2} \geq 0 \text {, } \\
& k=1,2, \cdots, K \text {. }
\end{aligned}
$$

In particular, given an $\boldsymbol{R}^{*}$ to problem (9), $\left(\boldsymbol{R}^{*} \bar{t}, \bar{t}, \bar{t}_{1}\right)$ is an optimal solution to (10), where

$$
\bar{t}=\left(\sum_{k=1}^{K} \max _{\boldsymbol{a}_{k} \in \mathcal{A}_{k}} \delta_{k} \boldsymbol{a}_{k}^{\dagger} \boldsymbol{R}^{*} \boldsymbol{a}_{k}+\sigma^{2}\right)^{-1}
$$

and $\bar{t}_{1}=\min _{\boldsymbol{a}_{0} \in \mathcal{A}_{0}} \delta_{0} \boldsymbol{a}_{0}^{\dagger} \boldsymbol{R}^{*} \boldsymbol{a}_{0}$. Conversely, assuming that $\left(\overline{\boldsymbol{R}}^{*}, t^{*}, t_{1}^{*}\right)$ is an optimal solution to $(10), \boldsymbol{R}^{*} / t^{*}$ is an optimal solution to (9).

Finally, it is worth pointing out that, for the case of no steering vector mismatches, the optimization problem (8) can be recast as

$$
\max _{\boldsymbol{R} \in \mathcal{R}_{u}} \frac{\delta_{0} \boldsymbol{a}_{0}^{\dagger} \boldsymbol{R} \boldsymbol{a}_{0}}{\sum_{k=1}^{K} \delta_{k} \boldsymbol{a}_{k}^{\dagger} \boldsymbol{R} \boldsymbol{a}_{k}+\sigma^{2}},
$$

which can be efficiently computed by related SDP technique [20].

\section{Constrained waveform design with known $R$}

In this section, we consider the waveform design problem by minimizing approximation error accounting for constant modulus and similarity constraints. Finally, we provide CA [21] to design the transmit waveform by exploiting the obtained $\boldsymbol{R}$.

\subsection{Constant modulus and similarity constraints}

In practical applications, the synthesized waveform should be unimodular (i.e., constant modulus) due to the limit of non-linear radar amplifiers. Hence, we 
here enforce the modulus of each element of $\boldsymbol{s}_{n}$, $n=1,2, \cdots, N_{T}$ to be constant, i.e., $\left|\boldsymbol{s}_{n}(m)\right|=\sqrt{c}$, $n=1,2, \ldots, N_{T}, m=1,2, \ldots, M$.

Additionally, we enforce $N_{T}$ different similarity constraints on the emitting waveforms, which employs a known code as a benchmark allowing the designed code to enjoy some good ambiguity characteristics of the known code, namely

$$
\left\|\boldsymbol{s}_{n}-\boldsymbol{s}_{n 0}\right\|_{\infty} \leq \xi_{n}, n=1,2, \cdots, N_{T},
$$

where $\boldsymbol{s}_{n 0} \in \mathbb{C}^{M}$ is the reference sequence vector at the $n$ th transmission interval and $\xi_{n}$ is a real parameter ruling the extent of the similarity. Without loss of generality, we assume the same similarity parameter $\xi$ (i.e., $\xi=\xi_{1}=$ $\cdots=\xi_{N_{T}}$ ) $[20,22,23]$, on the sought transmit waveform.

\subsection{Constrained waveform design problem}

In order to approximate a desired waveform covariance matrix $\boldsymbol{R}$ computed by (9), based on LS approach, thus the waveform synthesis problem under constant modulus and similarity constraints can be expressed as,

$$
\left\{\begin{array}{cl}
\min _{\boldsymbol{S}} & \left\|\boldsymbol{S S}^{\dagger}-\boldsymbol{R}\right\|^{2} \\
\text { s.t. } & \left\|\boldsymbol{s}_{n}-\boldsymbol{s}_{n 0}\right\|_{\infty} \leq \xi_{n}, n=1,2, \cdots, N_{T}, \\
& \left|\boldsymbol{s}_{n}(m)\right|=\sqrt{c}, n=1,2, \ldots, N_{T}, \\
& m=1,2, \cdots, M,
\end{array}\right.
$$

where $\boldsymbol{S}=\left[\boldsymbol{s}_{1}, \boldsymbol{s}_{2}, \cdots, \boldsymbol{s}_{N_{T}}\right]^{T} \in \mathbb{C}^{N_{T} \times M}$.

\subsection{Waveform synthesis algorithm}

In this subsection, we study the design procedure of transmit signal. Specifically, after some algebraic manipulations about similarity constraint [22] and according to [21], the waveform synthesis problem can be equivalent to

$$
\left\{\begin{array}{cl}
\min _{\boldsymbol{S}, \boldsymbol{U}} & \left\|\boldsymbol{S}-\sqrt{M} \boldsymbol{R}^{\frac{1}{2}} \boldsymbol{U}\right\|^{2} \\
\text { s.t. } & \boldsymbol{U} \boldsymbol{U}^{\dagger}=\boldsymbol{I}_{N_{T}}, \\
& \arg \boldsymbol{s}_{n}(m) \in\left[\gamma_{n m}, \gamma_{n m}+\delta_{n}\right], \\
& \left|\boldsymbol{s}_{n}(m)\right|=\sqrt{c}, n=1,2, \ldots, N_{T}, m=1,2, \ldots, M,
\end{array}\right.
$$

where $\boldsymbol{S}=\left[\boldsymbol{s}_{1}, \boldsymbol{s}_{2}, \cdots, \boldsymbol{s}_{N_{T}}\right]^{T}, \boldsymbol{U} \in \mathbb{C}^{N_{T} \times M}$ is an arbitrary unitary matrix demanding $\boldsymbol{U} \boldsymbol{U}^{\dagger}=\boldsymbol{I}_{N_{T}}$, and $\gamma_{n m}=$ $\arg s_{0 n}(m)-\arccos \left(1-\xi^{2} / 2\right), \delta_{n}=2 \arccos \left(1-\xi^{2} / 2\right)$ with $\xi=\frac{\xi_{n}}{\sqrt{c}}$. In particular, we can observe that for $\xi=0$, the designed $s_{n}$ is identical to known $s_{0 n}$, whereas the similarity constraint boils down to only the constant modulus constraint when $\xi=2$.

In particular, here we resort to CA to solve (15). Precisely, given a $\boldsymbol{S}$, the closed-form solution to (15) is $\boldsymbol{U}=$ $\boldsymbol{U}_{l} \boldsymbol{U}_{r}^{\dagger}$, where both $\boldsymbol{U}_{l} \in \mathbb{C}^{N_{T} \times N_{T}}$ and $\boldsymbol{U}_{r} \in \mathbb{C}^{M \times N_{T}}$ are unitary matrixes demanding $\sqrt{M} \boldsymbol{R}^{\frac{1}{2}} \boldsymbol{S}=\boldsymbol{U}_{l} \boldsymbol{\Lambda} \boldsymbol{U}_{r}^{\dagger}$ with $\boldsymbol{\Lambda} \in \mathbb{H}^{N_{T}}$ being a diagonal matrix. The detailed derivation can be found in [21]. Exploiting the estimated $\boldsymbol{U}$, we can find the nearest matrix to approximate $\sqrt{M} \boldsymbol{R}^{\frac{1}{2}} \boldsymbol{U}$. Specifically, with given a $\boldsymbol{U},(15)$ associated to the $m$ th variables of $\boldsymbol{s}_{n}$ is expressed as

$$
\begin{cases}\min _{\boldsymbol{s}_{n}(m)} & \left\|\boldsymbol{s}_{n}(m)-z_{n m}\right\|^{2} \\ \text { s.t. } & \arg \boldsymbol{s}_{n}(m) \in\left[\gamma_{n m}, \gamma_{n m}+\delta_{n}\right], \\ & \left|\boldsymbol{s}_{n}(m)\right|=\sqrt{c},\end{cases}
$$

with $z_{n m}$ is the $(n, m)$ th entry of $\sqrt{M} \boldsymbol{R}^{\frac{1}{2}} \boldsymbol{U}$. After some algebraic manipulations, (16) can be transformed as

$$
\begin{cases}\max _{s_{n}(m)} & \Re\left(\boldsymbol{s}_{n}(m) z_{n m}\right\} \\ \text { s.t. } & \arg \boldsymbol{s}_{n}(m) \in\left[\gamma_{n m}, \gamma_{n m}+\delta_{n}\right], \\ & \left|\boldsymbol{s}_{n}(m)\right|=\sqrt{c} .\end{cases}
$$

Further, the above problem is equivalent to

$$
\left\{\begin{array}{l}
\max _{\varphi_{n m}} \cos \left(\varphi_{c}+\varphi_{n m}\right) \\
\text { s.t. } \varphi_{n m} \in\left[\gamma_{n m}, \gamma_{n m}+\delta_{n}\right],
\end{array}\right.
$$

where $\varphi_{n m}$ and $\varphi_{c}$ are the phases of $\boldsymbol{s}_{n}(m)$ and $z_{n m}^{*}$, respectively. Hence, the optimization solution of (18) can be obtained by

$$
\varphi_{n m}^{*}=-\varphi_{c},-\varphi_{c} \in\left[\gamma_{n m}, \gamma_{n m}+\delta_{n}\right] ;
$$

otherwise, the optimal solution $\varphi_{n m}^{*}$ is given

$\varphi_{n m}^{*}= \begin{cases}\gamma_{n m}+\delta_{n} & \cos \left(\varphi_{c}+\gamma_{n m}+\delta_{n}\right) \geq \cos \left(\varphi_{c}+\gamma_{n m}\right) \\ \gamma_{n m} & \cos \left(\varphi_{c}+\gamma_{n m}+\delta_{n}\right)<\cos \left(\varphi_{c}+\gamma_{n m}\right) .\end{cases}$

Based on the above discussion, we can perform the same procedure to obtain the remaining variables among $\boldsymbol{S}$. Finally, the CA procedure involved in designing $S$ and $\boldsymbol{U}$ is summarized as Algorithm 1. It is worth pointing out that the total computational complexity of CA is related with the iteration number, the size of $\boldsymbol{S}$. In particular, each iteration requires to handle a singular value decomposition (SVD) of $N_{T} \times M$ dimension matrix with corresponding to computational complexity $O\left(M N_{T}^{2}+N_{T}^{3}\right)$ [24].

\section{Numerical results}

In this section, we evaluate the performance of proposed design technique of robust waveform focusing on a uniform linear array (ULA) transmit elements with $N_{T}=16$ assuming an inter-element spacing $d_{t}=\lambda / 2$. In particular, we consider the orthogonal linear frequency modulation (LFM) as the reference waveform $\boldsymbol{s}_{0}$ [20]. Specifically, the $\left(n_{t}, k\right)$ th entry of the reference $S^{(0)}$ is given by

$$
\boldsymbol{S}^{(0)}\left(n_{t}, m\right)=\exp \left\{j 2 \pi n_{t}(m-1) / N\right\} \exp \left\{j \pi(m-1)^{2} / N\right\},
$$

where $n_{t}=1,2, \cdots, N_{T}$ and $m=1,2, \cdots, M$. We suppose that the target is located at $\theta_{0}=0^{\circ}$ with power $\sigma_{0}^{2}=25 \mathrm{~dB}$. As to the Gaussian white noise, we set the 


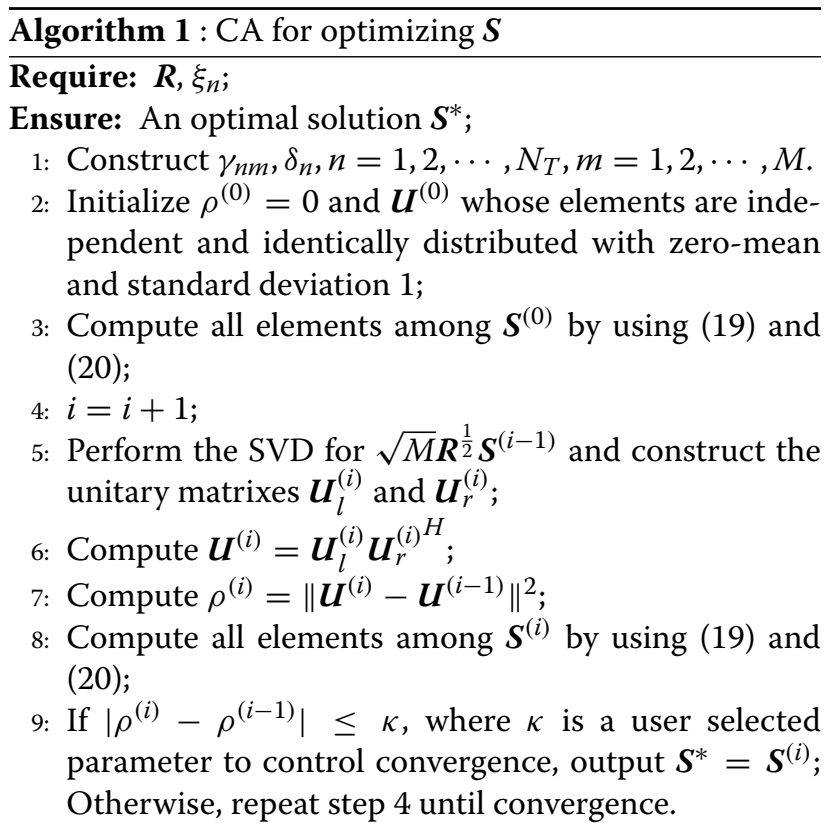

noise variance to $\sigma_{v}^{2}=0 \mathrm{~dB}$. The nominal ULA steering direction is given by

$$
\overline{\boldsymbol{a}}(\theta)=\frac{1}{\sqrt{N_{T}}}\left[1, e^{j 2 \pi \frac{d_{T}}{\lambda} \sin \theta}, \ldots, e^{j 2 \pi \frac{d_{T}}{\lambda}\left(N_{T}-1\right) \sin \theta}\right]^{T} .
$$

Additionally, we consider $c=1, \boldsymbol{B}_{k 1}=\boldsymbol{I}_{N_{T}}, \boldsymbol{b}_{k 1}=$ $\overline{\boldsymbol{a}}\left(\theta_{k}\right), b_{k 1}=1, u_{k 1}=\epsilon, \boldsymbol{B}_{k 2}=\boldsymbol{I}_{N_{T}}, \boldsymbol{b}_{k 2}=0, b_{k 2}=-1$, $u_{k 2}=0, k=0,1, \cdots, K$. To solve the convex optimisation problem (10), we resort to the CVX toolbox [25]. Finally, we consider the exit condition $\kappa=10^{-4}$ for Algorithm 1 , i.e.,

$$
\left|\rho^{(n)}-\rho^{(n-1)}\right| \leq 10^{-4}
$$

Hereafter, for any fixed waveform covariance matrix $\boldsymbol{R}$, the worst-case SINR $\rho^{\mathrm{wr}}$ can be obtained by

$$
\rho^{\mathrm{wr}}=\frac{\min _{\boldsymbol{a}_{0} \in \mathcal{A}_{0}} \delta_{0} \boldsymbol{a}_{0}^{\dagger} \boldsymbol{R} \boldsymbol{a}_{0}}{\sum_{k=1}^{K} \max _{\boldsymbol{a}_{k} \in \mathcal{A}_{k}} \delta_{k} \boldsymbol{a}_{k}^{\dagger} \boldsymbol{R} \boldsymbol{a}_{k}+\sigma^{2}},
$$

where the numerator and denominator of (23) can be efficiently computed by related SDP technique [19], respectively.

\subsection{Non-uniform point-like clutter}

In this subsection, we consider a non-uniform point-like clutter scenario where the angles and corresponding powers of seven interfering sources are reported in Table 1.

Figure 1 shows the worst-case SINR behavior versus uncertain parameter $\epsilon$ considering the non-robust and robust design of waveform covariance matrix, respectively. Note that the curve of non-robust case is plotted
Table 1 The angles and corresponding powers of seven interfering sources

\begin{tabular}{llllllll}
\hline Angle (deg) & -20 & 5 & 35 & 60 & 70 & -30 & -15 \\
Power (dB) & 20 & 20 & 30 & 25 & 28 & 30 & 10 \\
\hline
\end{tabular}

based on the obtained $\boldsymbol{R}$ according to (8) when no steering vector mismatches are foreseen at the design stage. These curves both decrease as the uncertain parameter $\epsilon$ grows up, implying that the steering vector mismatches indeed impair the detection performance. Interestingly, the robust design exhibits an improvement gain of SINR than that of non-robust design. In particular, the higher $\epsilon$, the gap of SINR between robust design and non-robust design becomes larger and larger.

In the following, based on the $\boldsymbol{R}$ computed by SDPrelated technique, we will focus on the waveform synthesis under constant modulus and similarity constraints by exploiting Algorithm 1. Specifically, we fix the uncertainly parameter $\epsilon=0.04$ for the following simulations. In addition, the approximation error $\eta$ between the obtained $\hat{\boldsymbol{R}}$ using Algorithm 1 and $\boldsymbol{R}$ is computed by

$$
\eta=\|\hat{\boldsymbol{R}}-\boldsymbol{R}\| \text {. }
$$

Figure 2 depicts the approximation error $\eta$ behavior versus iteration number considering different similarity levels for $M=20$. Notice that these obtained curves are averaged results of 100 statistic independent trials. As expected, the $\eta$ decreases with the increasing iteration number. Besides, the higher similarity level $\xi$, the lower $\eta$ is achieved due to the feasible set of optimization problem (15) becomes larger and larger.

Next, we analyze the impact of similarity parameters and sample length $M$ on the approximation error $\eta$ and SINR. In particular, we plot both the $\eta$ and SINR (dB) behaviors versus sample length $M$ considering $\xi=$ $0.3, .7,1.1,1.7,2$ in Fig. 3a, b, respectively. Notice that the black line in Fig. 3b represents the theoretical value without the synthetic loss of SINR. Results highlight that both the approximation error and SINR are associated with both similarity parameter $\xi$ and sample length $M$. Precisely, as $\xi$ and $M$ increases, the better approximation between $\hat{\boldsymbol{R}}$ and $\boldsymbol{R}$ can be achieved, which leads to higher gains of SINR. This is a reasonable behavior since the more degree of freedom (DOF) of waveform at design stage is obtained. In particular, when $M \geq 220$, the curve of SINR at $\xi=2$ overlaps with the theoretical curve showing that the constant modulus constraint causes no loss of SINR in the correspondence of the analysed parameters. However, it is worth pointing out that a loss of SINR can be observed due to the introduction of the similarity constraint. For example, we have the SINR gain of $2.8 \mathrm{~dB}$ from the black line in Fig. 3b, whereas the SINR becomes $2.2 \mathrm{~dB}$ at $\xi=1.7$. Besides, interestingly, for large $M$, both 


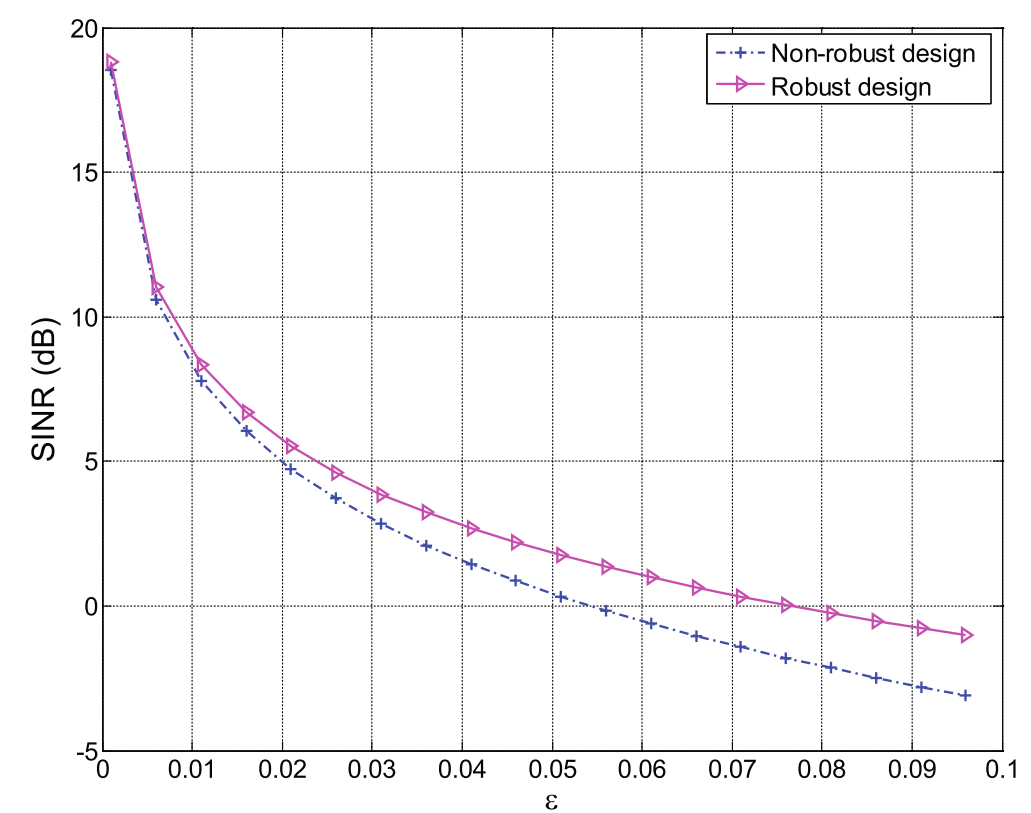

Fig. 1 The worst-case SINR (dB) behavior versus uncertain parameter $\epsilon$ in non-uniform clutter environment considering the non-robust and robust design of waveform covariance matrix

an increasing trend for SINR and a reducing trend for $\eta$ show unremarkable in Fig. 3b. This performance behavior provides a foundation for how to choose $M$ at waveform design stage. Finally, it is worth highlighting that a tradeoff should be considered between similarity level, sample length, and SINR.

\subsection{Uniform clutter}

In this subsection, we consider a uniform clutter scenario where we select $\left[-10^{\circ}, 50^{\circ}\right]$ as clutter region which is uniformly discretized with a grid size $1^{\circ}$. In particular, for each azimuth clutter bin, we consider a clutter-to-noise ratio (CNR) of $30 \mathrm{~dB}$.

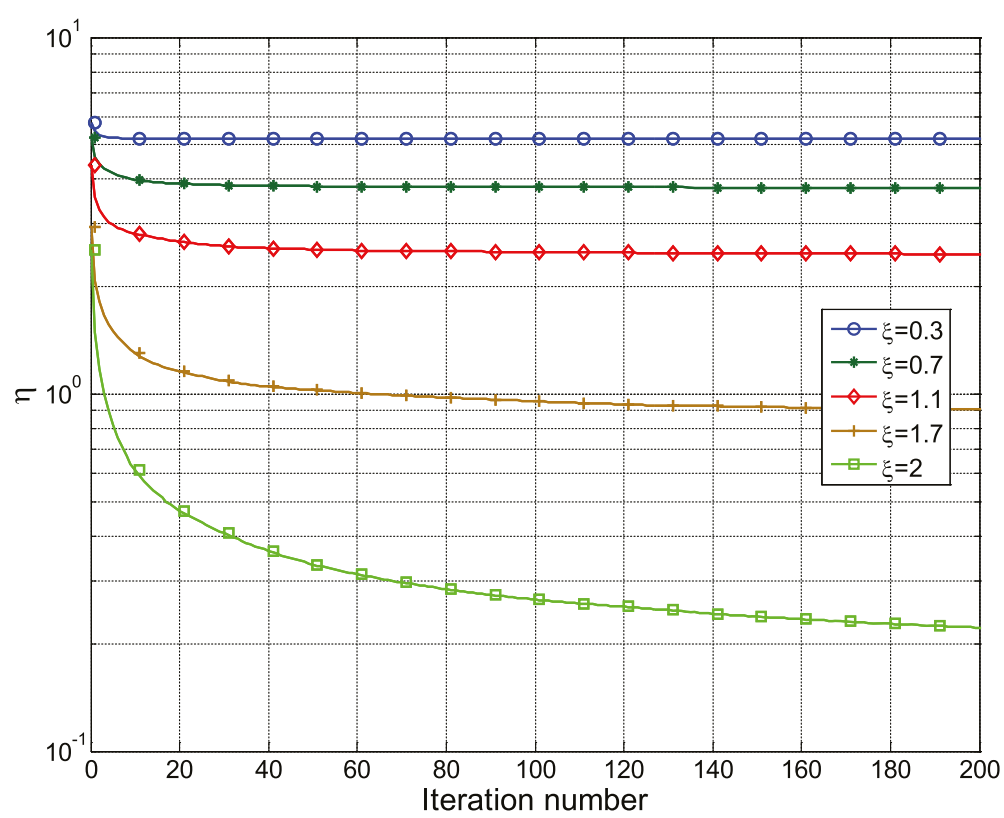

Fig. 2 The approximation error $\eta$ behavior versus iteration number in non-uniform point-like clutter environment for $\xi=0.3,0.7,1.1,1.7,2, M=20$ 


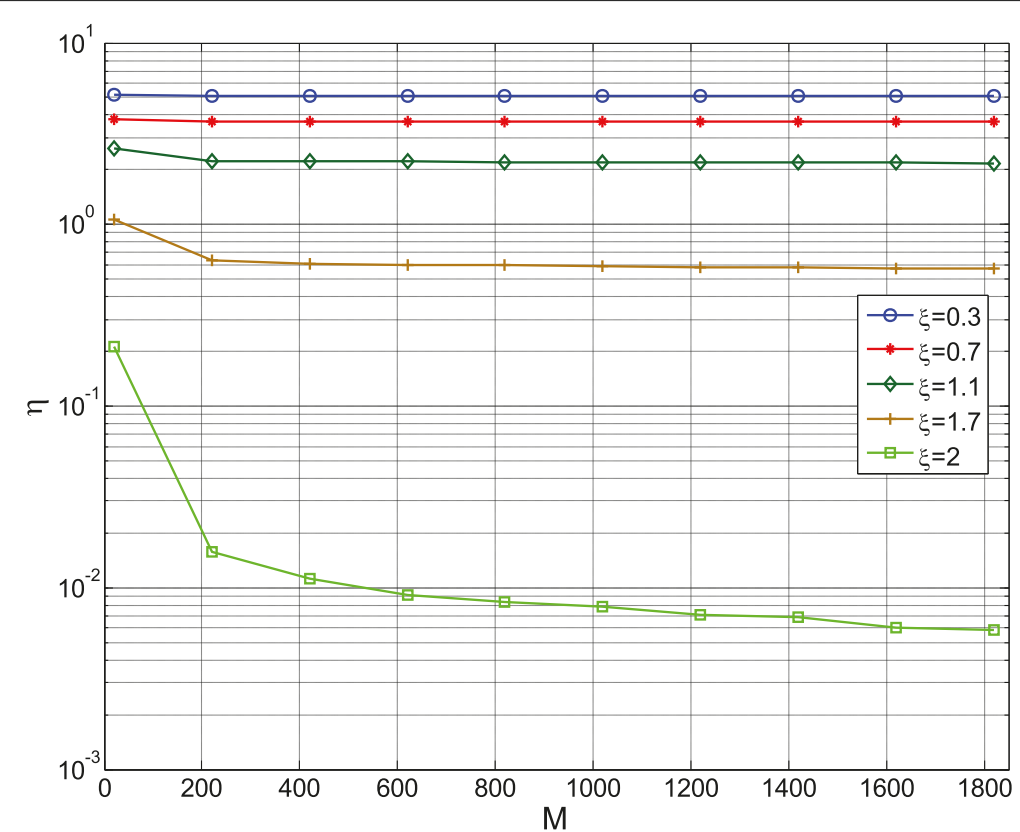

(a)

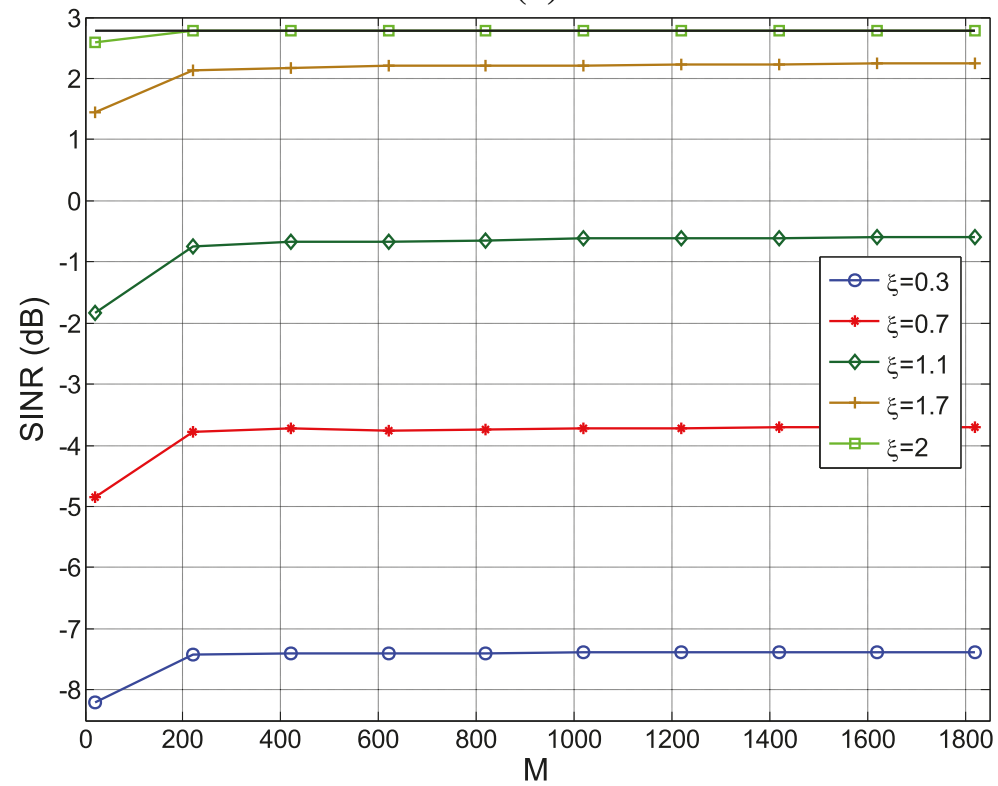

(b)

Fig. 3 The approximation error $\eta$ and SINR (dB) behaviors versus sample length $M$ considering $\xi=0.3,0.7,1.1,1.7,2$ in non-uniform point-like clutter environment, the black line representing the theoretical value of SINR, a $\eta, \mathbf{b} \operatorname{SINR}$

In Fig. 4, the worst-case SINR behavior considering the non-robust and robust design of waveform covariance matrix is plotted versus uncertain parameter $\epsilon$. The similar observation as Fig. 1 can be obtained. Indeed, the increasing $\epsilon$, both the non-robust design and robust design experience worse and worse SINR values due to the enlargement of mismatches between actual steering vectors and nominal ones. Again, it can be found that the robust design ensures a enhanced worst case performance in comparison with that of non-robust design. Finally, it is also interesting to notice that the gap between curves of non-robust design and robust design becomes larger and larger with the improvement of $\epsilon$.

In Fig. 5, the approximation error $\eta$ behavior, averaged over 100 independent trials of Algorithm 1, is plotted versus iteration number for $\xi=0.3,0.7,1.1,1.7,2, M=20$. Again, the smaller $\eta$ can be obtained as the increasing of 


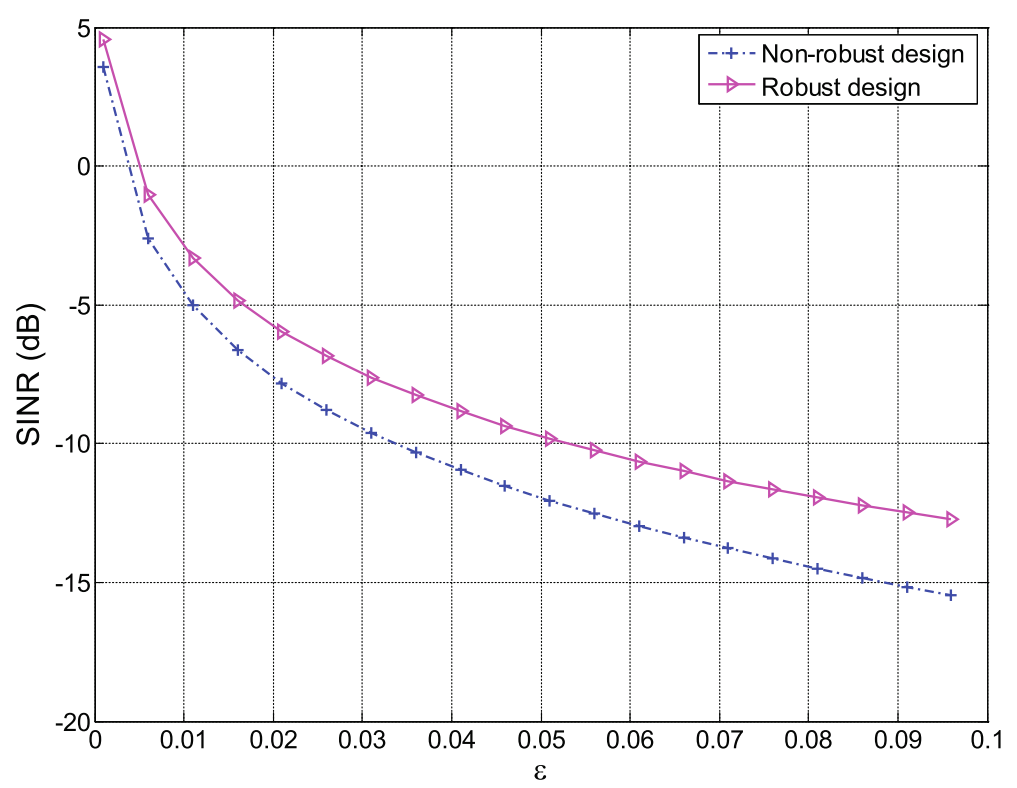

Fig. 4 The worst-case SINR (dB) behavior versus uncertain parameter $\epsilon$ in uniform clutter environment considering the non-robust and robust design of waveform covariance matrix

iteration number and $\xi$, revealing the better approximation between $\hat{\boldsymbol{R}}$ and $\boldsymbol{R}$.

In Fig. 6, the approximation error $\eta$ and SINR (dB) behaviors are plotted versus sample length $M$ considering $\xi=0.3,0.7,1.1,1.7,2$. Notice that the obtained curves are averaged results of 100 independent trials of Algorithm 1 and the black line in Fig. 6b stands for the theoretical value without the synthetic loss of SINR. Again, we observe that the higher $\xi$, the lower $\eta$ and the better SINR can be reached since the enlargement of the feasible sets of (15). In particular, we also find that both constant modulus and similarity constraints result in a loss of SINR. Specifically, we see the theoretical value of SINR is about $-8.7 \mathrm{~dB}$ from the black line in Fig. 6b, whereas it becomes about -9.9 and $-9.4 \mathrm{~dB}$ for $\xi=1.7$ and 2, respectively. Besides, both an increasing trend for SINR and an opposite trend for $\eta$ can be obtained as $M$ increases, whereas these trends are not apparent for large $M$. As a consequence, in practice,

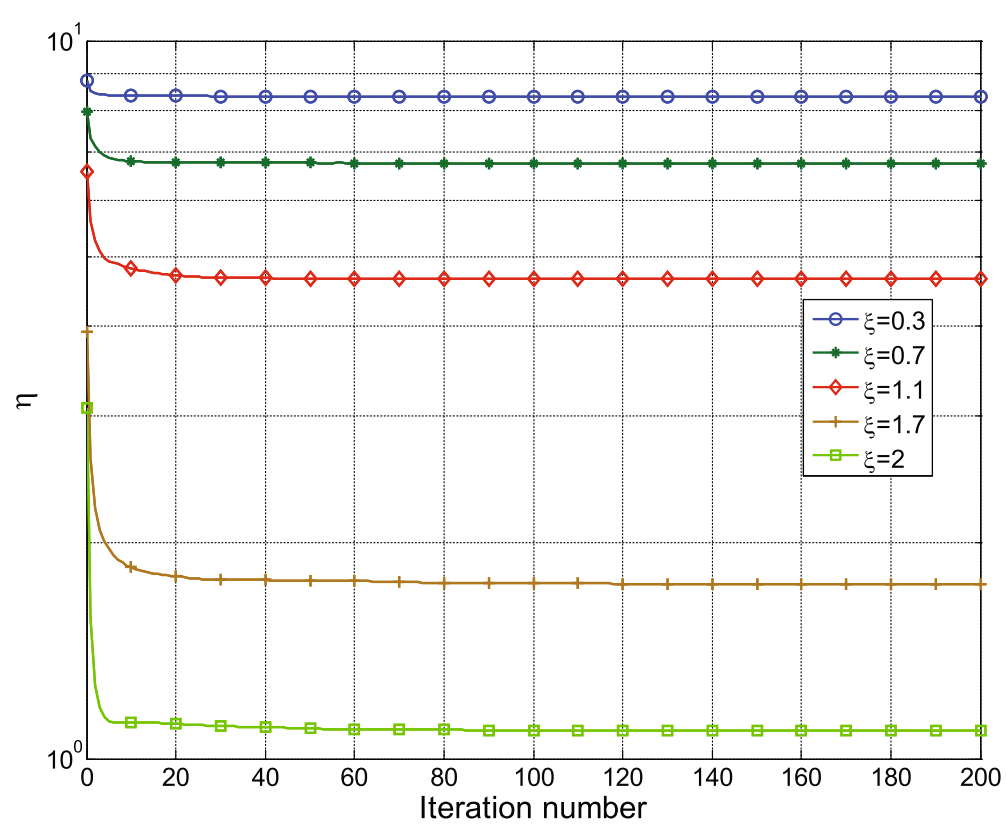

Fig. 5 The approximation error $\eta$ behavior versus iteration number in uniform clutter environment for $\xi=0.3,0.7,1.1,1.7,2, M=20$ 


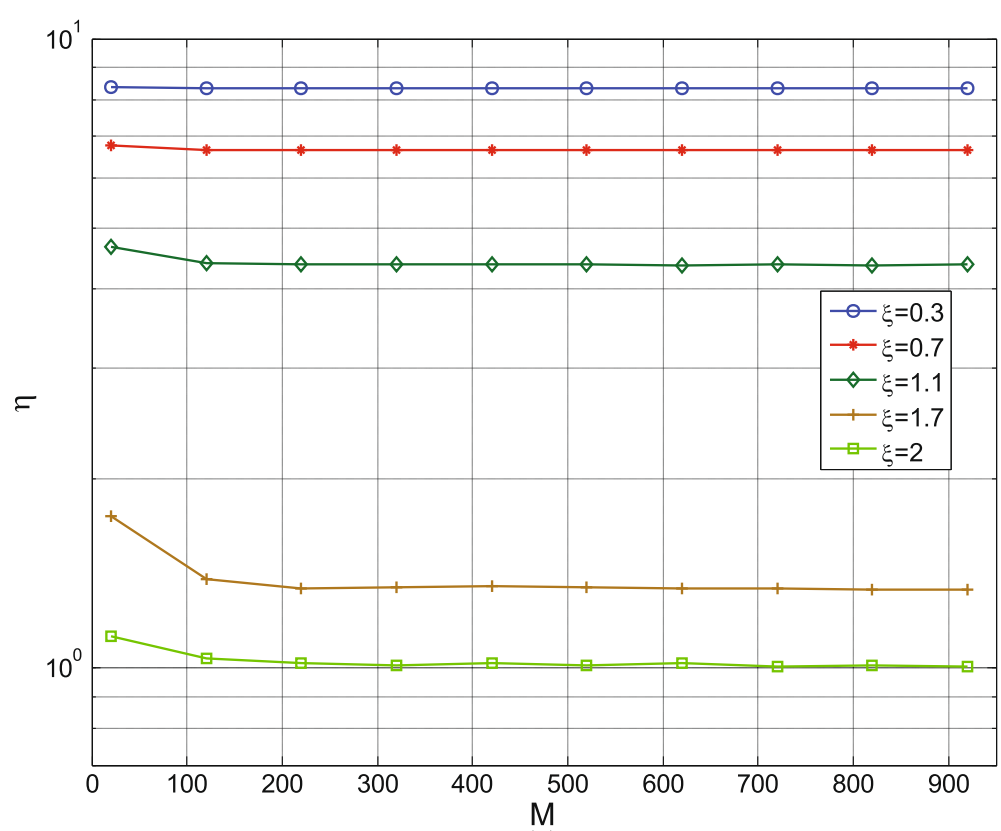

(a)

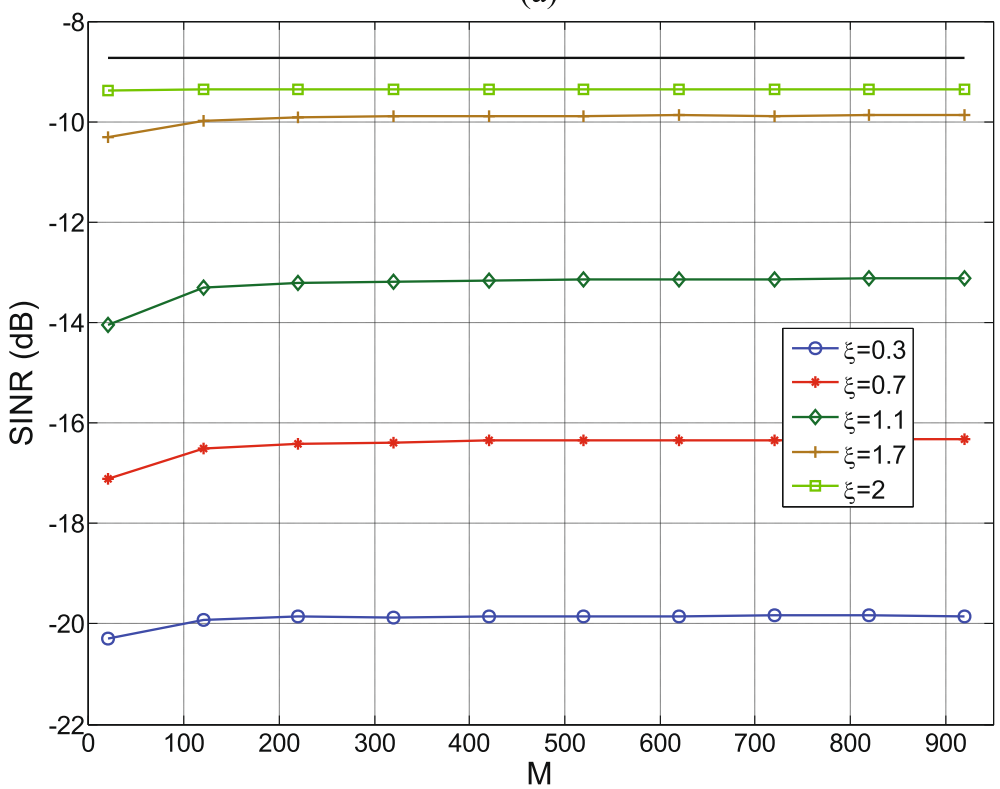

(b)

Fig. 6 The approximation error $\eta$ and SINR (dB) behaviors versus sample length $M$ considering $\xi=0.3,0.7,1.1,1.7,2$ in uniform clutter environment, the black line representing the theoretical value of SINR, a $\eta$, $\mathbf{b}$ SINR

we should reasonably consider the selection of sample length as well as the similarity parameter.

\section{Conclusions}

In this paper, we have considered the robust design of MIMO radar waveform under practical constraints for enhancing the targets delectability in the presence of signal-dependent interferences. Summarizing,

- We have optimized the waveform covariance matrix to improve the worst-case SINR against the uncertain sets of steering vector under a uniform power constraint through SDP-related technique. Based on LS approach, we have designed the MIMO waveform to approximate the desired waveform covariance matrix by using $\mathrm{CA}$ accounting for constant modulus and similarity constraints.

- We have provided numerical simulations to assess the performance of the proposed procedure. Results that the proposed algorithm enjoys the ability of ensuring an improved worst-case performance. We 
also observed that the approximation error decreases with the improvement of similarity level and sample length. As a consequence, we should choose reasonably the parameters of similarity level and sample length according to practical requirements.

Possible future research tracks might concern the extension of the proposed framework to account for both uncertain sets of angle and Doppler frequency of targets and interferes $[15,26]$, as well as the joint design of the transmit signal and receive filter for MIMO radar [20].

\section{Acknowledgements}

This work was supported by the National Natural Science Foundation of China under Grants 61201276, 61178068, and 61301266, the Fundamental Research Funds of Central Universities under Grants ZYGX2012Z001, ZYGX2013J012, ZYGX2014J013, and ZYGX2014Z005, the Chinese Postdoctoral Science Foundation under Grant 2014M550465, and by the Program for New Century Excellent Talents in University under Grant A1098524023901001063.

\section{Competing interests}

The authors declare that they have no competing interests.

\section{Author details}

${ }^{1}$ School of Electronic Engineering, University of Electronic Science and Technology of China, 2006 Xi Yuan Ave, West Hi-tech Zone, Chengdu, China. 2Elettronica S.p.A., Via Tiburtina Valeria km 13,700, 00131, Roma, Italy. ${ }^{3}$ Ente di Formazione Professionale Maxwell, via G. A. Campano, 103/105, I-80145, Napoli, Italy.

Received: 1 August 2016 Accepted: 16 December 2016

Published online: 04 January 2017

\section{References}

1. Ml Skolnik, Radar Handbook, 2nd Edition, (New York: McGraw-Hill, 1991)

2. SP Sira, Y Li, A Papandreou-Suppappola, D Morrell, D Cochran, M Rangaswamy, Waveform-agile sensing for tracking. IEEE Signal Process. Mag. 26(1), 53-64 (2009)

3. ZQ Luo, WK Ma, AC So, Y Ye, S Zhang, Semidefinite relaxation of quadratic optimization problems. IEEE Signal Process. Mag. 27(3), 20-34 (2010)

4. A De Maio, Y Huang, M Piezzo, A Doppler robust max-min approach to radar code design. IEEE Trans. Signal Process. 58(9), 4943-4947 (2010)

5. A De Maio, Y Huang, M Piezzo, S Zhang, F Alfonso, Design of optimized radar codes with a peak to average power ratio constraint. IEEE Trans. Signal Process. 59(6), 2683-2697 (2011)

6. MM Naghsh, M Soltanalian, P Stoica, M Modarres-Hashemi, A De Maio, A Aubry, A Doppler robust design of transmit sequence and receive filter in the presence of signal-dependent interference. IEEE Trans. Signal Process. 62(4), 772-785 (2014)

7. A Aubry, A De Maio, MM Naghsh, Optimizing radar waveform and Doppler filter bank via generalized fractional programming. IEEE J. Sel. Topics Signal Process. 9(8), 1387-1399 (2015)

8. SM Karbasi, A Aubry, A De Maio, HM Bastani, Robust transmit code and receive filter design for extended targets in clutter. IEEE Trans. Signal Process. 63(8), 1965-1976 (2015)

9. B Tang, J Tang, in 2016 IEEE International Conference on Acoustics, Speech and Signal Processing (ICASSP). Robust waveform design of wideband cognitive radar for extended target detection, (Shanghai, 2016), pp. 3096-3100

10. Y Yang, RS Blum, Minimax robust MIMO radar waveform design. IEEE J. Sel. Topics Signal Process. 1(1), 147-155 (2007)

11. P Stoica, J Li, Y Xie, On probing signal design for MIMO radar. IEEE Trans. Signal Process. 55(8), 4151-4161 (2007)

12. B Jiu, H Liu, D Feng, Z Liu, Minimax robust transmission waveform and receiving filter design for extended target detection with imprecise prior knowledge. Signal Process. 92(1), 210-218 (2012)

13. N Shariati, D Zachariah, M Bengtsson, in 2014 IEEE International Conference on Acoustics, Speech and Signal Processing (ICASSP). Minimum sidelobe beampattern design for $\mathrm{MIMO}$ radar systems: a robust approach, (Florence, 2014), pp. 5312-5316

14. H Wang, B Pei, Y Bai, in Signal Processing, Communications and Computing (ICSPCC) 2014 IEEE International Conference on. Robust waveform design for MIMO-STAP with imperfect clutter prior knowledge, (Guilin, 2014), pp. 578-581

15. SM Karbasi, A Aubry, V Carotenuto, MM Naghsh, HM Bastani, Knowledge-based design of space-time transmit code and receive filter for a multiple-input-multiple-output radar in signal-dependent interference. IET Radar Sonar Navig. 9(8), 1124-1135 (2015)

16. W Zhu, J Tang, Robust design of transmit waveform and receive filter for colocated MIMO radar. IEEE Signal Process. Lett. 22(11), 2112-2116 (2015)

17. A Aubry, A De Maio, A Farina, M Wicks, Knowledge-aided (potentially cognitive) transmit signal and receive filter design in signal-dependent clutter. IEEE Trans. Aerosp. Electron. Syst. 49(1), 93-117 (2013)

18. H Wang, G Liao, J Li, W Guo, Robust waveform design for MIMO-STAP to improve the worst-case detection performance. EURASIP J. Adv. Signal Process. 2013(1), 1 (2013)

19. A Aubry, A De Maio, Y Huang, MIMO Radar beampattern design via PSL/ISL optimization. IEEE Trans. Signal Process. 64(15), 3955-3967 (2016)

20. G Cui, H Li, M Rangaswamy, MIMO radar waveform design with constant modulus and similarity constraints. IEEE Trans. Signal Process. 62(2), 343-353 (2014)

21. P Stoica, J Li, XZhu, Waveform synthesis for diversity-based transmit beampattern design. IEEE Trans. Signal Process. 56(6), 2593-2598 (2008)

22. A De Maio, S De Nicola, Y Huang, Z Luo, S Zhang, Design of phase codes for radar performance optimization with a similarity constraint. IEEE Trans. Signal Process. 57(2), 610-621 (2009)

23. A Aubry, A De Maio, M Piezzo, A Farina, M Wicks, Cognitive design of the receive filter and transmitted phase code in reverberating environment. IET Radar Sonar Navig. 6(9), 822-833 (2012)

24. GH Golub, CFW Loan, Matrix Computations, 4rd Edition. (MD: The Johns Hopkins University Press, Baltimore, 2013)

25. M Grant, S Boyd, CVX package . http://www.cvxr.com/cvx.r, Accessed Feb 2012

26. X Yu, G Cui, L Kong, V Carotenuto, in 2016 IEEE Radar Conference. Space-time transmit code and receive filter design for colocated MIMO radar, (Philadelphia, 2016), pp. 1-6

\section{Submit your manuscript to a SpringerOpen ${ }^{\circ}$ journal and benefit from:}

- Convenient online submission

- Rigorous peer review

- Immediate publication on acceptance

- Open access: articles freely available online

- High visibility within the field

- Retaining the copyright to your article

Submit your next manuscript at $>$ springeropen.com 